\title{
ON THE WIENER CRITERION AND QUASILINEAR OBSTACLE PROBLEMS
}

\author{
JUHA HEINONEN AND TERO KILPELÄINEN
}

\begin{abstract}
We study the Wiener criterion and variational inequalities with irregular obstacles for quasilinear elliptic operators $A, A(x, \nabla u) \cdot \nabla u \approx|\nabla u|^{p}$, in $\mathbf{R}^{n}$. Local solutions are continuous at Wiener points of the obstacle function; if $p>n-1$, the converse is also shown to be true.

If $p>n-1$, then a characterization of the thinness of a set at a point is given in terms of $A$-superharmonic functions.
\end{abstract}

1. Introduction. In this paper we study the Wiener criterion and variational inequalities with irregular obstacles for quasilinear elliptic operators.

We assume throughout that $\psi: \mathbf{R}^{n} \rightarrow(-\infty, \infty)$ is a bounded Borel function, called an obstacle, and that $A: \mathbf{R}^{n} \times \mathbf{R}^{n} \rightarrow \mathbf{R}^{n}$ is an elliptic (nonlinear) operator with $A(x, h) \cdot h \approx|h|^{p}, 1<p \leq n$; for the precise assumptions on $A$, see (1.5)(1.9). A function $u$ is said to be a local solution to the obstacle problem at the point $x_{0} \in \mathbf{R}^{n}$ if there is an open neighborhood $\Omega$ of $x_{0}$ such that

$$
\left\{\begin{array}{l}
u \text { is in the Sobolev space } W_{p}^{1}(\Omega) ; \\
u \geq \psi p \text {-quasieverywhere in } \Omega ; \\
\int_{\Omega} A(x, \nabla u) \cdot \nabla \varphi d x \geq 0 \\
\text { whenever } \varphi \in W_{p, 0}^{1}(\Omega) \text { and } \varphi \geq \psi-u p \text {-quasieverywhere in } \Omega .
\end{array}\right.
$$

The precise meaning of " $p$-quasieverywhere" is explained below.

Further, if $u-v \in W_{p, 0}^{1}(\Omega)$, then $u$ is said to be a solution to the obstacle problem with the boundary values $v$ in $\Omega$.

In this paper we make an additional contribution to the regularity problem: what are the minimal conditions on $\psi$ which ensure that $u$ is continuous at $x_{0}$ ?

In the case of the Laplacian, i.e. $A(x, h)=h$, this problem was completely settled by J. Frehse and U. Mosco, see [FM1, FM2, Mo1, Mo2]. They have shown that the solution $u$ is continuous at $x_{0}$ if and only if $x_{0}$ is a so-called Wiener point of $\psi$. In a nonlinear case it was proved by J. H. Michael and W. P. Ziemer [MZ] that a similar condition is sufficient. Starting with ideas of P. Lindqvist and O. Martio [LM] we show that if $p>n-1$, then the Wiener criterion given in [MZ] is also necessary for the continuity of the solution of (1.1), see Theorem 1.16. Especially, the problem will be fully solved in dimension two.

If $\psi$ is nonnegative and supported on a compact subset $E$ of a bounded open set $\Omega$, then there is a unique nonnegative superharmonic function $u$ "vanishing" on the

Received by the editors August 4, 1987.

1980 Mathematics Subject Classification (1985 Revision). Primary 35J85; Secondary 31C05.

Key words and phrases. Wiener criterion, obstacle problem, $A$-superharmonic functions.

The first author was supported in part by Deutsche Forschungsgemeinschaft. 
boundary $\partial \Omega$, lying quasieverywhere above the obstacle $\psi$ in $\Omega$, and minimizing the Dirichlet integral among all admissible functions; in the language of potential theory the solution $u$ is the balayage of $\psi$. The continuity in this case was first studied by $\mathrm{H}$. Lewy and G. Stampacchia [LS]: if $\psi$ is continuous on $E$, then one arrives at the classical Wiener criterion. See also [CK, KS, Mo2].

It is important to note that the Perron approach to the unilateral Dirichlet problem pertains in more general situations; we shall indeed study the problem (1.1) within a general framework of nonlinear potential theory.

Before stating our main results we introduce some notation.

We assume throughout that $\Omega$ is a bounded open set in $\mathbf{R}^{n}, n \geq 2$, and $D \Subset \Omega$ means that $\bar{D}$, the closure of $D$, is compact in $\Omega$. If $B=B(x, r)$ is an open ball and $\sigma>0$, then $\sigma B=B(x, \sigma r)$. The integral averages are marked as

$$
f_{E} f d x=\frac{1}{|E|} \int_{E} f d x
$$

1.2. Condensers and capacities. Let $E$ be a subset of an open set $G \subset \mathbf{R}^{n}$. The (outer) $p$-capacity, $1<p \leq n$, of the condenser $(E, G)$ is defined as

$$
\operatorname{cap}_{p}(E, G)=\inf _{U \supset E \text { open }} * \operatorname{cap}_{p}(U, G)
$$

where, for any set $F \subset G$,

$$
{ }_{*} \operatorname{cap}_{p}(F, G)=\sup _{K \subset F \text { compact }} \inf _{u \in W(K, G)} \int_{G}|\nabla u|^{p} d x,
$$

and $W(K, G)=\left\{u \in C_{0}^{\infty}(G): u=1\right.$ in $\left.K\right\}$. It is standard that for a Borel set $E$,

$$
\operatorname{cap}_{p}(E, G)={ }_{*} \operatorname{cap}_{p}(E, G) .
$$

A set $E$ is said to be of p-capacity zero, abbreviated $\operatorname{cap}_{p} E=0$, if $\operatorname{cap}_{p}(E \cap G, G)=0$ for each open $G \subset \mathbf{R}^{n}$. For a bounded set $E$, $\operatorname{cap}_{p} E=0$ if $\operatorname{cap}_{p}(E, G)=0$ for some, and hence for all, bounded $G$ with $E \subset G$. We say that a property holds $p$-quasieverywhere, abbreviated $p$-q.e. (or, if no confusion arises, simply q.e.), if it holds except on a set of $p$-capacity zero.

For a thorough discussion of variational capacities we refer to $[\mathbf{M 2}, \mathbf{R}]$.

1.3. Sobolev spaces. The elements in the Sobolev space $W_{p}^{1}(\Omega), 1<p \leq n$, are considered as equivalence classes of functions $u$ which are defined in $\Omega$ up to a set of $p$-capacity zero; more precisely, if $u \in W_{p}^{1}(\Omega)$, then the limit

$$
\lim _{r \rightarrow 0} f_{B(x, r)} u(y) d y=u(x)
$$

exists and defines $u$ p-quasieverywhere in $\Omega$, see e.g. [AMS, FZ, R]. The subspace $W_{p, 0}^{1}(\Omega)$ is the closure of $C_{0}^{\infty}(\Omega)$ in $W_{p}^{1}(\Omega)$.

We assume that the operator $A: \mathbf{R}^{n} \times \mathbf{R}^{n} \rightarrow \mathbf{R}^{n}$ satisfies the following assumptions for some numbers $1<p \leq n$ and $0<\alpha \leq \beta<\infty$ :

the function $x \mapsto A(x, h)$ is measurable for all $h \in \mathbf{R}^{n}$, and the function $h \mapsto A(x, h)$ is continuous for a.e. $x \in \mathbf{R}^{n}$; 
for all $h \in \mathbf{R}^{n}$ and a.e. $x \in \mathbf{R}^{n}$

$$
\begin{gathered}
A(x, h) \cdot h \geq \alpha|h|^{p}, \\
|A(x, h)| \leq \beta|h|^{p-1}, \\
\left(A\left(x, h_{1}\right)-A\left(x, h_{2}\right)\right) \cdot\left(h_{1}-h_{2}\right)>0
\end{gathered}
$$

whenever $h_{1} \neq h_{2}$, and

$$
A(x, \lambda h)=|\lambda|^{p-2} \lambda A(x, h)
$$

for all $\lambda \in \mathbf{R}, \lambda \neq 0$.

A function $u \in \operatorname{loc} W_{p}^{1}(\Omega)$ is a solution (a supersolution) of the equation

$$
\nabla \cdot A(x, \nabla u)=0
$$

in $\Omega$ if

$$
\int_{\Omega} A(x, \nabla u) \cdot \nabla \varphi d x=0 \quad(\geq 0)
$$

for all $\varphi \in C_{0}^{\infty}(\Omega)\left(\varphi \in C_{0}^{\infty}(\Omega)\right.$ and $\left.\varphi \geq 0\right)$. As is well known, every solution of (1.10) has a (locally) Hölder continuous representative; we call continuous solutions of (1.10) A-harmonic. It is important in our case that $\lambda u+\mu$ is A-harmonic whenever $\lambda, \mu \in \mathbf{R}$ and $u$ is A-harmonic.

A typical example of the operators satisfying (1.5)-(1.9) is the $p$-harmonic operator $A(x, h)=|h|^{p-2} h$; solutions of the corresponding equation

$$
\nabla \cdot(|\nabla u|)^{p-2} \nabla u=0
$$

are customarily called $p$-harmonic.

Hereafter, the number $p, 1<p \leq n$, and the operator $A$ are fixed. We shall explicitly point out where the restriction $p>n-1$ is needed.

1.12. Thin sets. A set $E$ in $\mathbf{R}^{n}$ is said to be $(p$ - $)$ thin at the point $x_{0}$ if

$$
\int_{0}^{1}\left(\frac{\operatorname{cap}_{p}\left(E \cap B\left(x_{0}, t\right), B\left(x_{0}, 2 t\right)\right)}{\operatorname{cap}_{p}\left(B\left(x_{0}, t\right), B\left(x_{0}, 2 t\right)\right)}\right)^{1 /(p-1)} \frac{d t}{t}<\infty .
$$

The concept of a thin set is fundamental in potential theory and, traditionally, it has many equivalent formulations. In nonlinear theory the converse Wiener criterion (1.13) has turned out to be the right one, see [HW]. Our second main result, Theorem 1.23, yields in a special case a characterization which, similarly to the classical theory, is explicitly connected to the solvability of the Dirichlet problem, see Remark 4.6.

1.14. Wiener points of the obstacle. Following J. Frehse and U. Mosco we say that a point $x_{0}$ is a $(p$-) Wiener point of $\psi$ if for every $\varepsilon>0$ the set

$$
E_{\varepsilon}=\left\{x \in \mathbf{R}^{n}: \psi(x) \geq \bar{\psi}\left(x_{0}\right)-\varepsilon\right\}
$$

is not $\left(p\right.$-)thin at $x_{0}$; here and for the remainder of the discussion

$$
\bar{\psi}\left(x_{0}\right)=\inf _{r>0} \underset{x \in B\left(x_{0}, r\right)}{p \text { ess sup }} \psi(x),
$$

the abbreviation " $p$-ess sup" being self-explanatory. This definition generalizes the classical Wiener criterion in a natural way and, as we shall see, provides a complete answer to the regularity problem at least when $p>n-1$.

We begin with the following theorem which was proved by J. H. Michael and W. P. Ziemer in a more general context [MZ]. 
1.15. THEOREM. If $x_{0}$ is a Wiener point of $\psi$, then each local solution of (1.1) is continuous at $x_{0}$.

The surprising thing is that highly irregular obstacles may still produce continuous solutions. For completeness, we give a short proof for Theorem 1.15 in $\S 4$.

Our principal result is the following.

1.16. THEOREM. Suppose that $p>n-1$ and that $x_{0}$ is not a Wiener point of $\psi$. Then there is a local solution of (1.1) which cannot be made continuous at $x_{0}$.

In building a discontinuous solution we exploit a geometric method used by O. Martio and P. Lindqvist in [LM]; for $p \leq n-1$ this method breaks down for the unfortunate reason that the $p$-capacity of a continuum may vanish if $p \leq n-1$.

We now want to make Theorem 1.16 more precise and recall the definition for $A$-superharmonic functions (see [HK1]):

A lower semicontinuous (l.s.c.) function $u: G \rightarrow \mathbf{R} \cup\{\infty\}, G$ is open in $\mathbf{R}^{n}$, is $A$ superharmonic if for each domain $D \Subset G$ and each $A$-harmonic function $h \in C(\bar{D})$, $h \leq u$ in $\partial D$ implies $h \leq u$ in $D$.

An $A$-superharmonic function need not be even locally in the Sobolev space $W_{p}^{1}$. The following proposition reveals the precise connection between $A$-superharmonic functions and supersolutions of (1.10); for the proof, see [HK1].

1.17. PROpOsition. If $u$ is $A$-superharmonic in $\Omega$ and if $u \in \operatorname{loc} W_{p}^{1}(\Omega)$, then $u$ is a supersolution of (1.10) and

$$
u(x)=\operatorname{ess} \liminf _{y \rightarrow x} u(y)
$$

for all $x \in \Omega$; moreover, every locally upper bounded $A$-superharmonic function is locally in $W_{p}^{1}(\Omega)$.

Conversely, if $u$ is a supersolution of (1.10), then there is a unique A-superharmonic representative of $u$, given by (1.18).

1.19. COMPARISON PRINCIPLE [HK1,3.7]. If $u$ and $-v$ are A-superharmonic in $\Omega$, if

$$
\limsup _{y \rightarrow x} v(y) \leq \liminf _{y \rightarrow x} u(y)
$$

for all $x \in \partial \Omega$ and if the left- and the right-hand sides are not simultaneously $\infty$ or $-\infty$, then $v \leq u$ in $\Omega$.

It turns out that one can develop a nonlinear potential theory where $A$-superharmonic functions form a similar basis as superharmonic functions in the classical theory. In this study intrinsic properties of supersolutions rather than nonlinear potentials have a central rôle, cf. e.g. $[\mathbf{H W}, \mathbf{M K}, \mathbf{M e}]$. This paper is a continuation of our earlier works [HK1 and HK2]; see also [L], and [GLM1, GLM2, LM] for $p=n$.

1.20. Balayage. We define a balayage of the obstacle $\psi$ as follows. Let

$$
\Phi_{\Omega}^{\psi}=\{u: u \text { is } A \text {-superharmonic in } \Omega \text { and } u \geq \psi \text { q.e. in } \Omega\},
$$

and let

$$
Q_{\Omega}^{\psi}=\inf \Phi_{\Omega}^{\psi}
$$


Since $\psi$ is bounded, then the family $\Phi_{\Omega}^{\psi}$ is uniformly lower bounded by (1.18), and using classical methods (see Proposition 2.3 below) yields that the balayage

$$
\widehat{Q}_{\Omega}^{\psi}(x)=\liminf _{y \rightarrow x} Q_{\Omega}^{\psi}(y)
$$

is $A$-superharmonic in $\Omega$. Moreover, a generalization of the fundamental convergence theorem, Proposition 2.3, implies

$$
\widehat{Q}_{\Omega}^{\psi} \geq \psi \quad \text { q.e. in } \Omega \text {. }
$$

Thus, $\widehat{Q}_{\Omega}^{\psi}=Q_{\Omega}^{\psi} \in \Phi_{\Omega}^{\psi}$. In brief, the balayage $\widehat{Q}_{\Omega}^{\psi}$ is the smallest $A$-superharmonic function lying quasieverywhere above the obstacle $\psi$ in $\Omega$. Observe that since $\Omega$ is bounded, then $\psi$ is bounded, and hence $\widehat{Q}_{\Omega}^{\psi}$ belongs to loc $W_{p}^{1}(\Omega)$ by Proposition 1.17 .

When no confusion results, we denote $\widehat{Q}^{\psi}=\widehat{Q}_{\Omega}^{\psi}$.

Now Theorem 1.16 can be viewed as a corollary to the following result.

1.22. THEOREM. If $x_{0}$ is a Wiener point of $\psi$, then $\widehat{Q}_{\Omega}^{\psi}$ is continuous at $x_{0}$ whenever $\Omega$ is a neighborhood of $x_{0}$.

Conversely, if $p>n-1$ and if $x_{0}$ is not a Wiener point of $\psi$, then there is a neighborhood $\Omega$ of $x_{0}$ such that $\widehat{Q}_{\Omega}^{\psi}$ is discontinuous at $x_{0}$.

Applying (1.21) and 1.22 we obtain a generalization for a well-known result of classical potential theory.

1.23. THEOREM. A set $E$ is thin at $x_{0}$ if and, for $p>n-1$, only if there is a neighborhood $\Omega$ of $x_{0}$ and $A$-superharmonic function $u$ in $\Omega$ such that

$$
\liminf _{\substack{x \rightarrow x_{0} \\ x \in E \backslash\left\{x_{0}\right\}}} u(x)>u\left(x_{0}\right) .
$$

1.25. Regular open sets. The property (1.24) is closely connected to the following open problem in nonlinear potential theory. Let $G$ be a bounded open set in $\mathbf{R}^{n}$. The boundary point $x_{0} \in \partial G$ is said to be regular if $\lim _{x \rightarrow x_{0}} h_{f}(x)=f\left(x_{0}\right)$ whenever $f \in C(\bar{G}) \cap W_{p}^{1}(G)$ and $h_{f}$ is the unique $A$-harmonic function in $G$ with $h_{f}-f \in W_{p, 0}^{1}(G)$. (If every boundary point is regular, we say that $G$ is regular.) It was proved by V. G. Maz'ya [M1] that if $\mathbf{R}^{n} \backslash G$ is not thin at $x_{0}$, then $x_{0}$ is regular; conversely, P. Lindqvist and O. Martio [LM] have shown that if $p>n-1$, then $\mathbf{R}^{n} \backslash G$ is not thin at regular points. The converse part for $p \leq n-1$ is not known to the authors. We indicate in Remark 4.6 that, for all $p \in(1, n],(1.24)$ would imply that a regular point is not a thin point.

We think that Theorem 1.23 is true for all $p \in(1, n]$ although we have not found a proof.

The paper is organized as follows. In $\S 2$ we provide preliminary results which may have some independent interest in nonlinear potential theory; we compare two slightly different definitions for balayage. In $\S 3$ we prove Theorem 1.16 and the necessity part of Theorem 1.22. In the final section, $\S 4$, Theorems 1.15 and 1.23 and the sufficiency part of Theorem 1.22 are established. In $\S 4$ we also show that $A$-superharmonic functions are finely continuous everywhere.

Throughout the paper we let $C, C_{1}, \ldots$, denote various constants which are not necessarily the same in all occurrences and, unless otherwise stipulated, depend at most on $n, p, \alpha$ and $\beta$. 


\section{Preliminary results. Let}

$R^{\psi}=R^{\psi}(\Omega ; A)=\inf \{u: u$ is $A$-superharmonic in $\Omega$ and $u \geq \psi$ everywhere in $\Omega\}$ and let

$$
\widehat{R}^{\psi}(x)=\widehat{R}^{\psi}(\Omega ; A)(x)=\liminf _{y \rightarrow x} R^{\psi}(y)
$$

In taking the balayage it is often important to know whether $\widehat{R}^{\psi}=\widehat{Q}^{\psi}$, that is, whether sets of zero capacity can be neglected. In the Laplacian case the equality $\widehat{R}^{\psi}=\widehat{Q}^{\psi}$ is well known (note that $\Omega$ is assumed to be bounded, and hence Greenian) but in general we have only been able to verify the following weaker result which, together with its corollary, is our main goal in this section.

2.1. LEMMA. If $\widehat{Q}^{\psi} \in W_{p}^{1}(\Omega)$, then $\widehat{R}^{\psi}=\widehat{Q}^{\psi}$.

2.2. COROLLARY. Suppose that $\psi$ is nonnegative and compactly supported in Л. Then $\widehat{R}^{\psi}=\widehat{Q}^{\psi}$.

As the proof of Lemma 2.1 shows, the annoying fact that the sum of two $A$ superharmonic functions is not $A$-superharmonic in general can be bypassed to some extent when one is dealing with functions in $W_{p}^{1}(\Omega)$.

We shall appeal to the fundamental convergence theorem which we state here for convenience.

2.3. PROPOSITION [HK2, THEOREM 6.1]. Let $F$ be a locally uniformly lower bounded family of A-superharmonic functions in $\Omega$. If $w=\inf F$ and $\hat{w}(x)=$ $\liminf _{y \rightarrow x} w(y)$, then $\hat{w}$ is A-superharmonic in $\Omega$ and $\hat{w}=w$ q.e. in $\Omega$.

2.4. A-superharmonic functions and solutions to obstacle problems. Let $\xi: \Omega \rightarrow$ $[-\infty, \infty]$ be any function and let $\theta \in W_{p}^{1}(\Omega)$ be so that $\theta \geq \xi$ q.e. in $\Omega$. We demonstrate the fundamental fact that there is a unique solution $u$ to the obstacle problem (1.1) with the obstacle $\xi$ and the boundary values $\theta$, and that $u$ may be chosen to be A-superharmonic in $\Omega$.

Observe that our definition for the Sobolev space $W_{p}^{1}(\Omega)$ is different than that in [HK1 and HK2], and therefore a slightly more careful analysis is needed.

First, since the operator $A$ defines a strictly monotone, coercive and continuous mapping from the space $L_{p}^{1}(\Omega)=\left\{u: \nabla u \in L^{p}(\Omega)\right\}$ onto its dual, see e.g. [M1], there always exists a unique solution $v \in W_{p}^{1}(\Omega)$ with given boundary values, cf. [KS, p. 87]. Further, it was shown in [HK1, 3.17] that there is an $A$ superharmonic function $u$ such that $u=v$ a.e. in $\Omega$. We show that $u$ is in $W_{p}^{1}(\Omega)$ (i.e. the approximate limit (1.4) exists q.e. in $\Omega$ ), it then follows that $u=v$ q.e. in $\Omega$, cf. e.g. [MK, Lemma 5.8]. Indeed, since the functions $u_{k}=\min (u, k)$ are locally bounded, they satisfy (1.4) everywhere in $\Omega$ by [HK1, (2.28) and 3.15] and, consequently, $u_{k} \in W_{p}^{1}(\Omega)$. Now $u_{k}$ is a Cauchy sequence in $W_{p}^{1}(\Omega)$ and it follows e.g. from [MK, Lemma 5.7] that $u_{k} \rightarrow u_{0} \in W_{p}^{1}(\Omega)$ q.e. in $\Omega$. Since $u_{k} \rightarrow u$ everywhere in $\Omega$, we obtain $u=u_{0}$ q.e. in $\Omega$. This establishes that $u \in W_{p}^{1}(\Omega)$, whence $u=v$ q.e. in $\Omega$ as desired.

2.5. REMARK. As remarked in 2.4, for a bounded $A$-superharmonic function $u$ (1.4) holds everywhere, i.e. every point is a Lebesgue point of $u$. We do not 
know whether this is true for all $A$-superharmonic functions. Observe that an $A$ superharmonic function need not be locally integrable if $1<p \leq 2 n /(n+1)$, see [L].

Next, we state an approximation lemma, required in the proof of 2.1.

Let $\psi_{j} \in W_{p}^{1}(\Omega)$ be a decreasing sequence of functions (i.e. $\psi_{j} \geq \psi_{j+1}$ q.e.) such that $\psi_{j} \rightarrow \psi_{0}$ in $W_{p}^{1}(\Omega)$, and let $v_{j} \in W_{p}^{1}(\Omega)$ be the $A$-superharmonic solution to the obstacle problem with the obstacle and boundary values $\psi_{j}$. It is obvious and easy to prove, see [HK1, Lemma 2.8], that $v_{j} \geq v_{j+1}$ in $\Omega$.

2.6. LEMMA. The limit function $v=\lim v_{j}$ is the solution to the obstacle problem with the obstacle and boundary values $\psi_{0}$.

Proof. Clearly $v \in L^{p}(\Omega)$. Moreover, since $v_{j}$ is the solution to the obstacle problem,

$$
\sup _{j} \int_{\Omega}\left|\nabla v_{j}\right|^{p} d x \leq \sup _{j} C \int_{\Omega}\left|\nabla \psi_{j}\right|^{p} d x<\infty
$$

by Hölder's inequality. Hence $\nabla v_{j} \rightarrow \nabla v$ weakly in $L^{p}(\Omega)$, and it follows that $v-\psi_{0} \in W_{p, 0}^{1}(\Omega)$. Since, obviously, $v \geq \psi_{0}$ q.e. in $\Omega$, it suffices to show that

$$
\int_{\Omega} A(x, \nabla v) \cdot \nabla \varphi d x \geq 0
$$

whenever $\varphi \in W_{p, 0}^{1}(\Omega)$ and $\varphi \geq \psi_{0}-v$ q.e. in $\Omega$.

To prove (2.7), fix an admissible $\varphi$. Writing $w_{j}=\psi_{j}-\psi_{0}$ yields $v+w_{j}-v_{j} \geq$ $\psi_{j}-v_{j}$ q.e., whence

$$
\begin{aligned}
0 \geq & \int_{\Omega}\left(A\left(x, \nabla v_{j}\right)-A(x, \nabla v)\right) \cdot\left(\nabla v-\nabla v_{j}\right) d x \\
= & \int_{\Omega} A\left(x, \nabla v_{j}\right) \cdot \nabla\left(v+w_{j}-v_{j}\right) d x-\int_{\Omega} A\left(x, \nabla v_{j}\right) \cdot \nabla w_{j} d x \\
& -\int_{\Omega} A(x, \nabla v) \cdot\left(\nabla v-\nabla v_{j}\right) d x \\
\geq & -\int_{\Omega} A\left(x, \nabla v_{j}\right) \cdot \nabla w_{j} d x-\int_{\Omega} A(x, \nabla v) \cdot\left(\nabla v-\nabla v_{j}\right) d x .
\end{aligned}
$$

Since $\nabla w_{j} \rightarrow 0$ in $L^{p}(\Omega)$ and since $\nabla v_{j} \rightarrow \nabla v$ weakly in $L^{p}(\Omega)$, the last two integrals tend to zero as $j \rightarrow \infty$, and it follows from [M1, Lemma 1] that $A\left(x, \nabla v_{j}\right) \rightarrow$ $A(x, \nabla v)$ weakly in $L^{p /(p-1)}(\Omega)$.

To complete the proof, let $\varphi_{j}=\varphi+v+w_{j}-v_{j}$. Then $\varphi_{j} \in W_{p, 0}^{1}(\Omega)$ and $\varphi_{j} \geq \psi_{j}-v_{j}$ q.e., whence

$$
\begin{aligned}
\int_{\Omega} A & \left(x, \nabla v_{j}\right) \cdot \nabla \varphi d x \\
& =\int_{\Omega} A\left(x, \nabla v_{j}\right) \cdot \nabla \varphi_{j} d x+\int_{\Omega} A\left(x, \nabla v_{j}\right) \cdot\left(\nabla \varphi-\nabla \varphi_{j}\right) d x \\
& \geq \int_{\Omega} A\left(x, \nabla v_{j}\right) \cdot\left(\nabla v_{j}-\nabla v\right) d x-\int_{\Omega} A\left(x, \nabla v_{j}\right) \cdot \nabla w_{j} d x .
\end{aligned}
$$

Since again the last two integrals tend to zero as $j \rightarrow \infty$, we obtain

$$
\int_{\Omega} A(x, \nabla v) \cdot \nabla \varphi d x=\lim _{j \rightarrow \infty} \int_{\Omega} A\left(x, \nabla v_{j}\right) \cdot \nabla \varphi d x \geq 0
$$

as desired. 
Proof OF Lemma 2.1. We abbreviate $\hat{Q}=\hat{Q}^{\psi}, \hat{R}=\hat{R}^{\psi}$. Since clearly $\hat{R} \geq \hat{Q}$ q.e., it suffices to show that $\hat{R} \leq \hat{Q}$. Let $E=\{x \in \Omega: \hat{Q}(x)<\psi(x)\}$. Then, by Proposition 2.3, $E$ is of $p$-capacity zero, and hence there is a nonnegative l.s.c. function $w \in W_{p}^{1}\left(\mathbf{R}^{n}\right)$ such that $w=\infty$ in $E$, cf. [HK2, Theorem 1.5]. Let $\psi_{j}=\hat{Q}+j^{-1} w$ and let $v_{j}$ be the solution to the obstacle problem with the obstacle and boundary values $\psi_{j} \in W_{p}^{1}(\Omega)$. By 2.4 we may assume that each $v_{j}$ is $A$-superharmonic, whence with Proposition 1.17

$$
\begin{aligned}
v_{j}(x) & =\underset{y \rightarrow x}{\operatorname{ess} \liminf _{j}} v_{j}(y) \geq \underset{y \rightarrow x}{\operatorname{ess} \liminf _{y}(y)} \\
& \geq \psi_{j}(x) \geq \psi(x)
\end{aligned}
$$

for all $x$ in $\Omega$ (note that $\psi_{j}$ is l.s.c.). Thus, $v=\lim v_{j} \geq \hat{R}$. On the other hand, since $\psi_{j} \rightarrow \hat{Q}$ in $W_{p}^{1}(\Omega)$, then $v=\hat{Q}$ q.e. by Lemma 2.6 and by the uniqueness of the solution, cf. Proposition 1.17. Hence, again by Proposition 1.17,

$$
\hat{Q}(x)=\operatorname{ess} \liminf _{y \rightarrow x} v(y) \geq \operatorname{ess} \liminf _{y \rightarrow x} \hat{R}(y)=\hat{R}(x)
$$

for all $x$ in $\Omega$. This completes the proof of Lemma 2.1.

PROOF OF COROLlary 2.2. In the light of Lemma 2.1 it suffices to show that $\hat{Q}=\hat{Q}^{\psi} \in W_{p}^{1}(\Omega)$. First note that as a locally bounded $A$-superharmonic function $\hat{Q}$ is locally in $W_{p}^{1}(\Omega)$ by Proposition 1.17. Next, choose a neighborhood $D$ of $\partial \Omega$ so that $\bar{D} \cap \operatorname{spt} \psi=\varnothing$ and that $\mathbf{R}^{n} \backslash D$ is not thin at any point of $\partial D$. It is not difficult to show (see [HK2, 3.1 and 3.2]) that $\hat{Q}$ is $A$-harmonic in $\Omega \backslash$ spt $\psi$, consequently in $D \cap \Omega$. Choose a sequence of domains $\Omega_{j}$ so that $\partial D \cap \Omega \subset \Omega_{j} \Subset \Omega_{j+1} \Subset \Omega$, $\cup \Omega_{j}=\Omega$, and choose a function $\varphi \in C(\Omega) \cap W_{p}^{1}(\Omega)$ so that $\varphi=\hat{Q}$ in $\partial D \cap \Omega$ and that spt $\varphi \Subset \Omega$. Let $h_{j}$ be the unique $A$-harmonic function in $D \cap \Omega_{j}$ with $h_{j}-\varphi \in W_{p, 0}^{1}\left(D \cap \Omega_{j}\right)$, see 1.25. Since $\hat{Q} \in W_{p}^{1}\left(D \cap \Omega_{j}\right), \hat{Q} \geq 0$ and $\hat{Q}=\varphi=h_{j}$ in $\partial D \cap \Omega_{j}=\partial D \cap \Omega$, the standard comparison principle, see [HK1, 2.7], yields $\hat{Q} \geq$ $h_{j+1} \geq h_{j}$ in $D \cap \Omega_{j}$. By Harnack's principle [HK1,3.3] the function $h=\lim h_{j}$ is $A$-harmonic in $D \cap \Omega$. Moreover,

$$
\int_{D \cap \Omega_{j}}\left|\nabla h_{j}\right|^{p} d x \leq C \int_{\Omega}|\nabla \varphi|^{p} d x
$$

so that $h \in W_{p}^{1}(D \cap \Omega)$. We show that $h=\hat{Q}$ in $D \cap \Omega$; this completes the proof. Clearly $h \leq \hat{Q}$, and to prove the converse, consider the function

$$
h^{\prime}= \begin{cases}\min (h, \hat{Q}) & \text { in } D \cap \Omega, \\ \hat{Q} & \text { in } \Omega \backslash D .\end{cases}
$$

Since $\lim _{y \rightarrow x} h(y)=\hat{Q}(x)$ for all $x \in \partial D \cap \Omega, h^{\prime}$ is l.s.c. in $\Omega$. It is then easy to check, see Lemma 3.7 below, that $h^{\prime}$ is indeed $A$-superharmonic in $\Omega$, whence $h^{\prime} \geq \hat{Q}$ in $\Omega$ by (1.21). Thus $h=\hat{Q}$ in $D \cap \Omega$ as required.

The last lemma of this section allows us to deduce Theorem 1.16 from Theorem 1.22 . 
2.8. LemMA. The function $\hat{Q}^{\psi}$ is a local solution of (1.1).

Proof. Fix $x_{0} \in \Omega$ and let $B=B\left(x_{0}, r\right) \Subset \Omega$. Then $\hat{Q}=\hat{Q}^{\psi} \in W_{p}^{1}(B)$ by Proposition 1.17. Let $u$ be the solution to the obstacle problem in $B$ with the obstacle $\psi$ and the boundary values $\hat{Q}$. By 2.4 we may assume that $u$ is $A$ superharmonic. Further, $\operatorname{since} \min (u, \hat{Q})-\hat{Q} \in W_{p, 0}^{1}(B)$, then $\hat{Q} \geq u$ a.e. in $B$, see [HK1, 2.8]. Proposition 1.17 then implies that $\hat{Q} \geq u$ everywhere in $B$. We show that $u \geq \hat{Q}$ everywhere in $B$; this establishes the desired conclusion.

Thus, let $\varphi_{j} \in C_{0}^{\infty}\left(\mathbf{R}^{n}\right)$ be an increasing sequence such that $\lim \varphi_{j}=\hat{Q}$ in $\partial B$. Let $h_{j} \in C(\bar{B})$ be the unique $A$-harmonic function in $B$ such that $h_{j}=\varphi_{j}$ in $\partial B$, see 1.25. Then $\min \left(u-h_{j}, 0\right) \in W_{p, 0}^{1}(B)$ and the comparison principle [HK1, 2.7] yields $u \geq h_{j}$ in $B$. This shows that $\liminf _{y \rightarrow x} u(y) \geq \hat{Q}(x)$ for all $x \in \partial B$, whence the function

$$
w= \begin{cases}\min (\hat{Q}, u) & \text { in } B, \\ \hat{Q} & \text { in } \Omega \backslash B\end{cases}
$$

is l.s.c. and, therefore, $A$-superharmonic in $\Omega$, see Lemma 3.7. Since $w \geq \psi$ q.e. in $\Omega$ by (1.21), then $u \geq w \geq \hat{Q}$ everywhere in $B$ as desired.

3. The proof of the necessity part of Theorem 1.22; the proof of Theorem 1.16. If $E \subset \Omega$, we then call the function $u=\hat{R}_{E}^{1}=\hat{R}^{\chi_{E}}(\Omega ; A)$ the $A$-potential of $E$ in $\Omega$; here the obstacle $\chi_{E}$ is the characteristic function of $E$, cf. $\S 2$.

We start with the following simple lemma.

3.1. LEMMA. If $K \subset \Omega$ is compact and if $\varphi \in C_{0}^{\infty}(\Omega)$ is such that $\varphi=1$ in a neighborhood of $K$, then the A-potential $u=\hat{R}_{K}^{1}$ is the A-harmonic function in $\Omega \backslash K$ with $u-\varphi \in W_{p, 0}^{1}(\Omega \backslash K)$.

ProOF. The lemma can be proved by using the comparison principle and an exhaustion argument basically as in the proof of Corollary 2.2. The details are left to the reader.

Next, we require two lemmas similar to [LM, 3.6 and 3.16]. We suppose that $K$ is a compact set in a ball $B=B\left(x_{0}, 2 r\right)$ and that $u=\hat{R}_{K}^{1}$ is the $A$-potential of $K$ in $B$.

3.2. Lemma. Let $0<\gamma<1$ and $E_{\gamma}=\{x \in B: u(x) \geq \gamma\}$. There is a constant $C_{1}=C_{1}(p, \alpha, \beta)>0$ such that

$$
\frac{1}{C_{1}} \gamma^{p-1} \operatorname{cap}_{p}\left(E_{\gamma}, B\right) \leq \operatorname{cap}_{p}(K, B) \leq C_{1} \gamma^{p-1} \operatorname{cap}_{p}\left(E_{\gamma}, B\right) .
$$

ProOF. Using Lemma 3.1 and Hölder's inequality yields

$$
\frac{1}{C} \operatorname{cap}_{p}(K, B) \leq \int_{B} A(x, \nabla u) \cdot \nabla u d x \leq C \operatorname{cap}_{p}(K, B),
$$


and, therefore, since $u \in W_{p}^{1}(B \backslash K)$ is a solution of $(1.10)$ in $B \backslash K$,

$$
\begin{aligned}
\operatorname{cap}_{p}\left(E_{\gamma}, B\right) & \leq \gamma^{-p} \int_{\{u<\gamma\}}|\nabla u|^{p} d x \leq C \gamma^{-p} \int_{\{u<\gamma\}} A(x, \nabla u) \cdot \nabla u d x \\
& =C \gamma^{1-p} \int_{B} A(x, \nabla u) \cdot \nabla \min (u / \gamma, 1) d x \\
& =C \gamma^{1-p} \int_{B} A(x, \nabla u) \cdot \nabla u d x \leq C \gamma^{1-p} \operatorname{cap}_{p}(K, B) .
\end{aligned}
$$

Similarly,

$$
\begin{aligned}
\operatorname{cap}_{p}\left(E_{\gamma}, B\right) & \geq C \gamma^{-p} \int_{\{u<\gamma\}} A(x, \nabla u) \cdot \nabla u d x \\
& \geq C \gamma^{-p}\left(\int_{B} A(x, \nabla u) \cdot \nabla u d x-(1-\gamma) \int_{\{u>\gamma\}} A(x, \nabla u) \cdot \nabla \frac{u-\gamma}{1-\gamma} d x\right) \\
& =C \gamma^{-p}\left(\int_{B} A(x, \nabla u) \cdot \nabla u d x-(1-\gamma) \int_{B} A(x, \nabla u) \cdot \nabla u d x\right) \\
& \geq C \gamma^{1-p} \operatorname{cap}_{p}(K, B) .
\end{aligned}
$$

3.4. LEMMA. Let $p>n-1$. There is a constant $C_{2}=C_{2}(n, p, \alpha, \beta)>0$ such that if

$$
\operatorname{cap}_{p}(K, B) / \operatorname{cap}_{p}\left(\frac{1}{2} B, B\right) \leq C_{2} \gamma^{p-1},
$$

then $u<\gamma$ on some sphere $S\left(x_{0}, \rho\right)=\partial B\left(x_{0}, \rho\right), r / 4 \leq \rho \leq r / 2$.

PROOF. Suppose that each sphere $S\left(x_{0}, \rho\right), r / 4 \leq \rho \leq r / 2$, meets the set $E_{\gamma}=\{x \in B: u(x) \geq \gamma\}, 0<\gamma<1$. Then, by the symmetrization (see e.g. [Sa]),

$$
\begin{aligned}
\operatorname{cap}_{p}\left(E_{\gamma}, B\right) & \geq \operatorname{cap}_{p}\left(\left[x_{0}+r e_{1} / 4, x_{0}+r e_{1} / 2\right], B\right) \\
& \geq C \operatorname{cap}_{p}\left(\frac{1}{2} B, B\right),
\end{aligned}
$$

where $e_{1}=(1,0, \ldots, 0)$, and for the last inequality see e.g. [M2, 9.1.2]. Thus, by $(3.3)$

$$
\gamma^{p-1} \leq C\left[\operatorname{cap}_{p}(K, B) / \operatorname{cap}_{p}\left(\frac{1}{2} B, B\right)\right]
$$

and the lemma follows.

3.5. REMARK. Lemma 3.4 is clearly false if $p \leq n-1$.

In practice it is more convenient to use a Wiener sum instead of the Wiener integral (1.13). Thus, suppose that $E \subset \mathbf{R}^{n}$ and that $x_{0} \in E$. Write $B_{j}=$ $B\left(x_{0}, 2^{-j}\right)$ and

$$
a_{j}=\frac{\operatorname{cap}_{p}\left(E \cap B_{j}, B_{j-1}\right)}{\operatorname{cap}_{p}\left(B_{j}, B_{j-1}\right)} .
$$

Then

3.6. LEMMA.

$$
\int_{0}^{1}\left(\frac{\operatorname{cap}_{p}\left(E \cap B\left(x_{0}, t\right), B\left(x_{0}, 2 t\right)\right)}{\operatorname{cap}_{p}\left(B\left(x_{0}, t\right), B\left(x_{0}, 2 t\right)\right)}\right)^{1 /(p-1)} \frac{d t}{t}<\infty
$$

if and only if $\sum_{j=1}^{\infty} a_{j}^{1 /(p-1)}<\infty$. 
PROOF. The details of the proof are left to the reader, see [LM, 3.15]. Indeed, the claim is a simple consequence of the fact that if $\delta>1$, then

$$
\begin{gathered}
\frac{1}{C} \operatorname{cap}_{p}\left(E \cap B\left(x_{0}, t\right), B\left(x_{0}, 2 t\right)\right) \leq \operatorname{cap}_{p}\left(E \cap B\left(x_{0}, t\right), B\left(x_{0}, \delta t\right)\right) \\
\leq C \operatorname{cap}_{p}\left(E \cap B\left(x_{0}, t\right), B\left(x_{0}, 2 t\right)\right),
\end{gathered}
$$

where $C$ depends on $\delta$ but neither on $E$ nor on $t$; these inequalities can be established by using an auxiliary Lipschitz stretching.

3.7. LEMMA. Suppose that $u$ and $v$ are A-superharmonic in $\Omega$ and that $G \subset \Omega$ is open. If the function

$$
w= \begin{cases}\min (u, v) & \text { in } G, \\ u & \text { in } \Omega \backslash G\end{cases}
$$

is l.s.c. in $\Omega$, then it is A-superharmonic in $\Omega$.

PROOF. The claim follows straightforwardly from the comparison principle 1.19 and from the definition for $A$-superharmonic functions.

PROOF OF THE NECESSITY PART IN THEOREM 1.22. Let $p>n-1$. Suppose that $x_{0}$ is not a Wiener point of $\psi$ and let $\varepsilon>0$ be such that the set

$$
E=E_{\varepsilon}=\left\{x \in \mathbf{R}^{n}: \psi(x) \geq \bar{\psi}\left(x_{0}\right)-\varepsilon\right\}
$$

is thin at $x_{0}$. Then fix $\varepsilon_{1}>0$ (we shall later choose $\varepsilon_{1}$ appropriately) and choose $R_{0}>0$ such that if $R_{j}=2^{-j} R_{0}$ and $B_{j}=B\left(x_{0}, R_{j}\right)$, then $\sum_{j=1}^{\infty} a_{j}^{1 /(p-1)}<\varepsilon_{1}$, where

$$
a_{j}=\frac{\operatorname{cap}_{p}\left(E \cap B_{j}, B_{j-1}\right)}{\operatorname{cap}_{p}\left(B_{j}, B_{j-1}\right)} \text {. }
$$

Next, choose open sets $U_{j} \subset B_{j}$ such that $E \cap B_{j} \subset U_{j}$ and that $\sum_{j=1}^{\infty} b_{j}^{1 /(p-1)}<$ $2 \varepsilon_{1}$, where

$$
b_{j}=\frac{\operatorname{cap}_{p}\left(U_{j}, B_{j-1}\right)}{\operatorname{cap}_{p}\left(B_{j}, B_{j-1}\right)} \text {. }
$$

Let $u_{j}=\hat{R}_{U_{j}}^{1}\left(B_{j-1} ; A\right)$ be the $A$-potential of $U_{j}$ in $B_{j-1}$. Then $u_{j}=1$ in $U_{j}$. Moreover, there is a sphere $S_{j}=\partial B\left(x_{0}, \rho_{j}\right) \subset \bar{B}_{j+1} \backslash B_{j+2}$ such that

$$
u_{j}<C_{3} b_{j}^{1 /(p-1)}=d_{j}
$$

on $S_{j}$; here $C_{3}=C_{3}(n, p, \alpha, \beta)>0$. To prove (3.8), fix $j$. Then choose an increasing sequence of compact sets $K_{i} \subset U_{j}$ such that $\bigcup_{i} K_{i}=U_{j}$ and that every point in $\partial K_{i}$ is regular for the open set $B_{j-1} \backslash K_{i}$. If $v_{i}=\hat{R}_{K_{i}}^{1}\left(B_{j-1} ; A\right)$, then clearly $\lim _{i \rightarrow \infty} v_{i}=$ $u_{j}$. On the other hand, by Lemma 3.4 , there is a sphere $S_{j}=\partial B\left(x_{0}, \rho_{j}\right) \subset$ $\bar{B}_{j+1} \backslash B_{j+2}$ such that

$$
v_{i} \leq C_{2}^{1 /(1-p)}\left(\frac{\operatorname{cap}_{p}\left(K_{i}, B_{j-1}\right)}{\operatorname{cap}_{p}\left(B_{j}, B_{j-1}\right)}\right)^{1 /(p-1)} \leq C_{2}^{1 /(1-p)} b_{j}^{1 /(p-1)},
$$

on $S_{j}$ and (3.8) follows.

We now choose $\varepsilon_{1}=1 / 4 C_{3}$ so that $\sum_{j=1}^{\infty} d_{j}<\frac{1}{2}$. 
Next, write $b=p$-ess $\sup _{B_{1}} \psi$ and $c=\bar{\psi}\left(x_{0}\right)-\varepsilon$. We are clearly free to assume that $b<\bar{\psi}\left(x_{0}\right)+\varepsilon / 2$. Next, let $\varphi=(\psi-c) /(b-c)$ and $\varphi_{1}=\max (0, \varphi)$. We show that $v=\hat{Q}_{B_{1}}^{\psi}$ is the required balayage, discontinuous at $x_{0}$; for this, it suffices to show that

$$
p \text { - ess } \liminf _{x \rightarrow x_{0}} v_{1}(x)<1 / 2
$$

where

indeed, (3.9) implies

$$
v_{1}=\hat{Q}_{B_{1}}^{\varphi_{1}} \geq \hat{Q}_{B_{1}}^{\varphi}=(v-c) /(b-c)
$$

$$
\liminf _{x \rightarrow x_{0}} v(x)<(b+c) / 2<\bar{\psi}\left(x_{0}\right)-\varepsilon / 4,
$$

while

$$
\limsup _{x \rightarrow x_{0}} v(x) \geq p \text { - ess } \limsup _{x \rightarrow x_{0}} v(x) \geq \bar{\psi}\left(x_{0}\right)
$$

by $(1.21)$.

To prove (3.9), first observe that since $0 \leq \varphi_{1} \leq 1$ and since $\varphi_{1}=0$ q.e. in $B_{1} \backslash U_{1}$, then $u_{1} \geq v_{1}$ in $B_{1}$. Thus, by (3.8), $v_{1}<d_{1}$ on $S_{1}=\partial B\left(x_{0}, \rho_{1}\right) \subset \bar{B}_{2} \backslash B_{3}$. If

$$
v_{2}=\frac{v_{1}-d_{1}}{1-d_{1}} \quad \text { and } \quad \varphi_{2}=\frac{\varphi_{1}-d_{1}}{1-d_{1}}
$$

then $v_{2}=\hat{Q}_{B_{1}}^{\varphi_{2}}$. Write

$$
w_{2}= \begin{cases}\min \left(v_{2}, u_{2}\right) & \text { in } B\left(x_{0}, \rho_{1}\right), \\ v_{2} & \text { in } B_{1} \backslash B\left(x_{0}, \rho_{1}\right) .\end{cases}
$$

Since $v_{2} \leq 0$ on $S_{1}=\partial B\left(x_{0}, \rho_{1}\right)$, the function $w_{2}$ is l.s.c. and hence $A$-superharmonic in $B_{1}$ by Lemma 3.7. On the other hand, $w_{2} \geq \varphi_{2}$ q.e. in $B_{1}$, whence $w_{2} \geq v_{2}$ in $B_{1}$, whence $u_{2} \geq v_{2}$ in $B\left(x_{0}, \rho_{1}\right)$; in particular, $v_{1}-d_{1}<u_{2}<d_{2}$ on $S_{2}=\partial B\left(x_{0}, \rho_{2}\right) \subset \bar{B}_{3} \backslash B_{4}$. Next, write

$$
v_{3}=\frac{v_{2}-d_{2}}{1-d_{2}} \quad \text { and } \quad \varphi_{3}=\frac{\varphi_{2}-d_{2}}{1-d_{2}} .
$$

Then $v_{3}=\hat{Q}_{B_{1}}^{\varphi_{3}}$. As above we infer that the function

$$
w_{3}= \begin{cases}\min \left(v_{3}, u_{3}\right) & \text { in } B\left(x_{0}, \rho_{2}\right), \\ v_{3} & \text { in } B_{1} \backslash B\left(x_{0}, \rho_{2}\right)\end{cases}
$$

is l.s.c. and hence $A$-superharmonic in $B_{1}$; in particular $v_{2}-d_{2}<d_{3}$ on $S_{3}=$ $\partial B\left(x_{0}, \rho_{3}\right) \subset \bar{B}_{4} \backslash B_{3}$. It follows that $v_{1}<d_{1}+d_{2}+d_{3}$ on $S_{3}$, and continuing in this way we obtain

$$
v_{1}<\sum_{j=1}^{k} d_{j} \leq \sum_{j=1}^{\infty} d_{j}<\frac{1}{2}
$$

on $S_{k}=\partial B\left(x_{0}, \rho_{k}\right) \subset \bar{B}_{k+1} \backslash B_{k}$. Thus, (3.9) follows.

The necessity part in Theorem 1.22 is then proved.

PROOF OF THEOREM 1.16. If $x_{0}$ is not a Wiener point of $\psi$ and if $p>n-1$, then, by Theorem 1.22 , there is a neighborhood $\Omega$ of $x_{0}$ such that the function 
$\hat{Q}=\hat{Q}_{\Omega}^{\psi}$ is discontinuous at $x_{0}$. It follows from Lemma 2.8 that $\hat{Q}$ is a local solution of (1.1), and if $v$ is any representative of $\hat{Q}$, it follows from (1.18) that

$$
\text { ess } \liminf _{x \rightarrow x_{0}} v(x)=\hat{Q}\left(x_{0}\right)<\operatorname{ess} \limsup _{x \rightarrow x_{0}} \hat{Q}(x)=\operatorname{ess} \limsup _{x \rightarrow x_{0}} v(x)
$$

so that $v$ cannot be continuous at $x_{0}$.

4. Thin sets and $A$-superharmonic functions. In this final section we prove Theorem 1.23 and the sufficiency part of Theorem 1.22; a short proof for Theorem 1.15 is also given. Furthermore, we show that $A$-superharmonic functions are finely continuous.

Let $E$ be a set in $\mathbf{R}^{n}$ and let $x_{0} \in \mathbf{R}^{n}$. Consider the three statements:

(A) There is an A-superharmonic function $u$ in a neighborhood $U$ of $x_{0}$ with

$$
\liminf _{\substack{x \rightarrow x_{0} \\ x \in E \backslash\left\{x_{0}\right\}}} u(x)>u\left(x_{0}\right) \text {. }
$$

(B) There are neighborhoods $U$ and $V$ of $x_{0}, V \Subset U$, and a nonnegative $A$ superharmonic function $u$ in $U$ such that

$$
\hat{R}_{E \cap V}^{u}\left(x_{0}\right)<u\left(x_{0}\right), \quad \text { where } \hat{R}_{E \cap V}^{u}=\hat{R}^{u \chi_{E \cap V}}(U ; A) .
$$

(C) $E$ is thin at $x_{0}$.

In the classical theory the statements (A), (B) and (C) are equivalent. In the nonlinear case we establish the equivalence when $p>n-1$; for $p \leq n-1$ we have only been able to prove the implications $(\mathrm{A}) \Rightarrow(\mathrm{B}) \Rightarrow(\mathrm{C})$.

\subsection{Proposition. (A) implies (B).}

ProOF. Since (B) is trivially true if $x_{0} \notin \overline{E \backslash\left\{x_{0}\right\}}$, we may assume $x_{0} \in \overline{E \backslash\left\{x_{0}\right\}}$. Let $u$ be the $A$-superharmonic function given in (A). We may assume that $u>0$ and that

$$
\liminf _{\substack{x \rightarrow x_{0} \\ x \in E \backslash\left\{x_{0}\right\}}} u(x)>1>u\left(x_{0}\right)
$$

Choose an open neighborhood $V$ of $x_{0}, V \Subset U$, such that $u>1$ in $(E \cap V) \backslash\left\{x_{0}\right\}=F$. Then

$$
1>u\left(x_{0}\right) \geq \hat{R}_{F}^{1}\left(x_{0}\right)=\hat{R}_{E \cap V}^{1}\left(x_{0}\right),
$$

where the last equality follows from Corollary 2.2. This proves (B).

To complete the chain, we require the following simple lemma.

4.2. LEMMA. If $E$ is a Borel set which is not thin at $x_{0}$, then there is a compact set $K \subset E \cup\left\{x_{0}\right\}$ such that $K$ is not thin at $x_{0}$.

If $E$ is thin at $x_{0}$, then there is an open neighborhood $U$ of $E \backslash\left\{x_{0}\right\}$ such that $U$ is thin at $x_{0}$.

PrOOF. We prove the second assertion only; the proof of the first assertion is even simpler and left to the reader (recall that for a Borel set $E$ in $G$

$$
\left.\operatorname{cap}_{p}(E, G)=\sup _{K \subset E \text { compact }} \operatorname{cap}_{p}(K, G)\right) \text {. }
$$


Thus, for each $j=1,2, \ldots$ choose an open set $U_{j} \subset B\left(x_{0}, 2^{-j}\right)=B_{j}$ such that $E \cap B_{j} \subset U_{j}$ and that

$$
a_{j}^{1 /(p-1)}=\left(\frac{\operatorname{cap}_{p}\left(E \cap B_{j}, B_{j-1}\right)}{\operatorname{cap}_{p}\left(B_{j}, B_{j-1}\right)}\right)^{1 /(p-1)} \geq\left(\frac{\operatorname{cap}_{p}\left(U_{j}, B_{j-1}\right)}{\operatorname{cap}_{p}\left(B_{j}, B_{j-1}\right)}\right)^{1 /(p-1)}-2^{-j} .
$$

We may clearly assume that $U_{j+1} \subset U_{j}$ and that $E \subset B_{1}$. Next, let $U=$ $\bigcup_{j}\left(U_{j} \backslash \bar{B}_{j+2}\right)$. Then $E \backslash\left\{x_{0}\right\} \subset U$. Further, $\operatorname{cap}_{p}\left(U \cap B_{1}, B_{0}\right) \leq \operatorname{cap}_{p}\left(B_{1}, B_{0}\right)$, and for $j=2,3, \ldots$

$$
\operatorname{cap}_{p}\left(U \cap B_{j}, B_{j-1}\right) \leq \operatorname{cap}_{p}\left(U_{j-1} \cap B_{j}, B_{j-1}\right) \leq C \operatorname{cap}_{p}\left(U_{j-1}, B_{j-2}\right),
$$

cf. Lemma 3.6. Hence

$$
\sum_{j=1}^{\infty}\left(\frac{\operatorname{cap}_{p}\left(U \cap B_{j}, B_{j-1}\right)}{\operatorname{cap}_{p}\left(B_{j}, B_{j-1}\right)}\right)^{1 /(p-1)} \leq C+C \sum_{j=2}^{\infty}\left(a_{j}^{1 /(p-1)}+2^{-j}\right)<\infty,
$$

and the claim follows from Lemma 3.6.

\subsection{Proposition. (B) implies (C).}

ProOF. Let $u, U$ and $V \Subset U$ be as in (B). We may assume that $U$ is bounded and regular and that $u>0$. By Choquet's topological lemma [D, p. 792] there is a decreasing sequence $w_{j}$ of nonnegative $A$-superharmonic functions in $U$ such that $w_{j}=u$ in $E \cap V$ and that for $w=\lim w_{j}$ it holds

$$
\hat{w}(x)=\liminf _{y \rightarrow x} w(y)=\hat{R}_{E \cap V}^{u}(x)
$$

for all $x \in U$. Considering the Borel set $E^{\prime}=\{x \in V: w(x)=u(x)\} \supset E$, we obtain

$$
\hat{R}_{E^{\prime}}^{u}\left(x_{0}\right) \leq \hat{w}\left(x_{0}\right)=\hat{R}_{E \cap V}^{u}\left(x_{0}\right)<u\left(x_{0}\right),
$$

and hence we may assume, initially, that $E$ is a Borel set. Further, by Corollary 2.2 , we may assume that $x_{0} \in E$.

Let us suppose that $E$ is not thin at $x_{0}$. Then, by Lemma 4.2 , there is a compact set $K \subset E \cap V$ such that $K$ is not thin at $x_{0}$. Let $\varphi_{j} \in C_{0}^{\infty}(U)$ be an increasing sequence such that $\lim \varphi_{j}=u$ in $K$ and let $h_{j}$ be the unique $A$-harmonic function in $U \backslash K$ with $h_{j}-\varphi_{j} \in W_{p, 0}^{1}(U \backslash K)$, see 1.25. Then $u \geq h_{j}$ in $U \backslash K$ : Fix $j$ and let $v \geq 0$ be $A$-superharmonic in $U$ with $v \geq u$ in $K$; then $v^{\prime}=\min \left(v, \sup h_{j}\right)$ is in $W_{p}^{1}(V)$ by Proposition 1.17. Since for every $\varepsilon>0$ the function $\min \left(v^{\prime}+\varepsilon-h_{j}, 0\right)$ is in $W_{p, 0}^{1}(U \backslash K)$, the comparison principle [HK1,2.7] implies $v^{\prime} \geq h_{j}$ in $U \backslash K$. Thus, we infer that $u \geq R_{K}^{u}=R^{u \chi K}(U ; A) \geq h_{j}$ in $U \backslash K$. Hence we obtain

$$
\begin{aligned}
\hat{R}_{K}^{u}\left(x_{0}\right) & =\sup _{r>0} \inf _{B\left(x_{0}, r\right)} R_{K}^{u} \\
& =\min \left(\liminf _{\substack{y \rightarrow x_{0} \\
y \in U \backslash K}} R_{K}^{u}(y), u\left(x_{0}\right)\right) \\
& \geq \min \left(\lim _{\substack{y \rightarrow x_{0} \\
y \in U \backslash K}} h_{j}(y), u\left(x_{0}\right)\right)=\varphi_{j}\left(x_{0}\right) ;
\end{aligned}
$$


the last equality results since $K$ is not thin at $x_{0}$, see 1.25 . Letting $j \rightarrow \infty$ yields

$$
u\left(x_{0}\right) \leq \hat{R}_{K}^{u}\left(x_{0}\right) \leq \hat{R}_{E \cap V}^{u}\left(x_{0}\right)<u\left(x_{0}\right)
$$

which is absurd. This completes the proof.

4.4. Proposition. If $p>n-1$, then (C) implies (A).

ProOF. Let $U$ be the open neighborhood of $E \backslash\left\{x_{0}\right\}$, given in Lemma 4.2. Then $U$ is thin at $x_{0}$. (We may clearly assume $x_{0} \subset \bar{U}$.) If $\psi=\chi_{U}$, the characteristic function of $U$, then for $0<\varepsilon<1$ the set

$$
E_{\varepsilon}=\left\{x \in \mathbf{R}^{n}: \psi(x) \geq \bar{\psi}\left(x_{0}\right)-\varepsilon=1-\varepsilon\right\}=U
$$

is thin at $x_{0}$. By Theorem 1.22 there is a neighborhood $\Omega$ of $x_{0}$ so that the balayage $\hat{Q}^{\psi}=\hat{Q}_{\Omega}^{\psi}$ is discontinuous at $x_{0}$. Since $\hat{Q}^{\psi} \leq 1, \hat{Q}^{\psi}\left(x_{0}\right)<1$. However, obviously $\hat{Q}^{\psi}=1$ in $U \cup \Omega$, whence

$$
\liminf _{\substack{x \rightarrow x_{0} \\ x \in E \backslash\left\{x_{0}\right\}}} \hat{Q}^{\psi}(x) \geq 1>\hat{Q}^{\psi}\left(x_{0}\right) .
$$

This proves (A) since $\hat{Q}^{\psi}$ is $A$-superharmonic.

It is known that functions in the Sobolev space $W_{p}^{1}$ are finely continuous $p$ quasieverywhere, see e.g. $[\mathbf{H W}, \mathbf{M e}]$. The next theorem says that an $A$-superharmonic function is finely continuous everywhere. For bounded supersolutions this was proved by J. H. Michael and W. P. Ziemer in [MZ]; our proof is a simple application of Propositions 4.1 and 4.3 above.

4.5. THEOREM. Let $u$ be A-superharmonic in $\Omega$ and let $x_{0} \in \Omega$. There is a set $E$ such that $E$ is thin at $x_{0}$ and that $\left.u\right|_{\Omega \backslash E}$ is continuous at $x_{0}$.

ProOF. If $u\left(x_{0}\right)=\infty$, we may choose $E=\varnothing$. Thus, suppose that $u\left(x_{0}\right)<\infty$. For each $j=1,2, \ldots$ let $E_{j}=\left\{x \in \Omega: u(x)-u\left(x_{0}\right)>1 / j\right\}$. Then, by Propositions 4.1 and $4.3, E_{j}$ is thin at $x_{0}$. Now it is easy to see that there is a decreasing sequence $r_{j} \rightarrow 0$ such that the set $E=\bigcup_{j}\left(E_{j} \cap B\left(x_{0}, r_{j}\right)\right)$ is thin at $x_{0}$, cf. [Me, Proposition 3.1]. Since $u$ is l.s.c., the theorem is thereby proved.

For completeness, we apply Theorem 4.5 and give a short proof for Theorem 1.15 (cf. [MZ]): Let $u \in W_{p}^{1}(\Omega)$ be a local solution of (1.1). By 2.4 we may assume that $u$ is nonnegative and $A$-superharmonic in $\Omega$. Let $E$ be a set as in Theorem 4.5, that is, $E$ is thin at $x_{0}$ and $\left.u\right|_{\Omega \backslash E}$ is continuous at $x_{0}$. Since for each $\varepsilon>0$ the set $F_{\varepsilon}=\left\{x \in \mathbf{R}^{n}: \psi(x) \geq \bar{\psi}\left(x_{0}\right)-\varepsilon\right\} \cap(\Omega \backslash E)$ is not thin at $x_{0}, \operatorname{cap}_{p}\left(B \cap F_{\varepsilon}, 2 B\right)>0$ for each ball $B=B\left(x_{0}, r\right)$, and it follows that $u\left(x_{0}\right) \geq \bar{\psi}\left(x_{0}\right)-\varepsilon$. Consequently, $u\left(x_{0}\right) \geq \bar{\psi}\left(x_{0}\right)$. Next, fix $\lambda>u\left(x_{0}\right)$. The estimate [HK1, (2.19)] yields, for $r>0$ small enough,

$$
\begin{aligned}
\underset{B\left(x_{0}, r\right)}{\operatorname{ess} \sup }(u-\lambda)^{+} & \leq C f_{B\left(x_{0}, 2 r\right)}(u-\lambda)^{+} d x \\
& =C f_{B\left(x_{0}, 2 r\right)}(u-\min (u, \lambda)) d x
\end{aligned}
$$

where $(u-\lambda)^{+}=\max (u-\lambda, 0)$. Since bounded $A$-superharmonic functions are approximately continuous by [HK1, (2.28)], the latter integral tends to zero as 
$r \rightarrow 0$. Thus, by (1.18),

$$
\limsup _{x \rightarrow x_{0}} u(x) \leq \lambda
$$

and letting $\lambda \rightarrow u\left(x_{0}\right)$ establishes the desired conclusion.

PROOF OF THE SUFFICIENCY PART IN THEOREM 1.22. The claim follows from Theorem 1.15 and Lemma 2.8 .

4.6. Concluding REMARKS. (a) Write $E=\mathbf{R}^{n} \backslash \Omega$ and let $x_{0} \in \partial \Omega$. In the light of the barrier characterization the regularity of the boundary point is a local property (this was discussed in [GLM2] for $p=n$, and the general case is similar). Consequently, one easily obtains that the condition (B) above implies that $x_{0}$ is not regular. To be precise, let $u$ be as in (B), $x_{0} \in V \Subset U$. Further, let $\varphi_{j} \in C_{0}^{\infty}(U)$ be an increasing sequence such that $\lim \varphi_{j}=u$ in $\partial(V \cap \Omega)$ and let $h_{j}$ be the unique $A$-harmonic function in $U \cap \Omega$ with $h_{j}-\varphi_{j} \in W_{p, 0}^{1}(U \cap \Omega)$. Then $h_{j} \leq \hat{R}_{E \cap V}^{u}$ in $U \cap \Omega$, see the proof of Proposition 4.3. If $x_{0}$ is regular, then

$$
\varphi_{j}\left(x_{0}\right)=\lim _{x \rightarrow x_{0}} h_{j}(x) \leq \hat{R}_{E \cap V}^{u}\left(x_{0}\right),
$$

and letting $j \rightarrow \infty$ yields $u\left(x_{0}\right) \leq \hat{R}_{E \cap V}^{u}\left(x_{0}\right)$, which is a contradiction.

We conclude that proving $(\mathrm{C}) \Rightarrow(\mathrm{A})$ (or $(\mathrm{C}) \Rightarrow(\mathrm{B})$ ) also when $p \leq n-1$ would completely solve the boundary regularity problem for quasilinear equations of the type (1.10).

(b) We have assumed throughout that the obstacle $\psi$ is bounded. It is however often useful to consider obstacles which may take values at infinity, cf. the discussion in 2.4. Are Theorems 1.15 and 1.22 true also if $\psi$ is unbounded?

\section{REFERENCES}

[AMS] N. Aronszajn, F. Mulla and P. Szeptycki, On spaces of potentials connected with $L^{p}$-classes, Ann. Inst. Fourier (Grenoble) 13 (1963), 211-306.

[CK] L. A. Caffarelli and D. Kinderlehrer, Potential methods in variational inequalities, J. Analyse Math. 37 (1980), 285-295.

[D] J. L. Doob, Classical potential theory and its probabilistic counterpart, Springer-Verlag, New York and Berlin, 1984.

[FZ] H. Federer and W. P. Ziemer, The Lebesgue set of a function whose partial derivatives are $p$-th power summable, Indiana Univ. Math. J. 22 (1972), 139-158.

[FM1] J. Frehse and U. Mosco, Sur la continuité ponctuelle des solutions locales faibles du problème d'obstacle, C. R. Acad. Sci. Paris Ser. A 295 (1982), 571-574.

[FM2] _ Wiener obstacles, Séminaire Collège de France, Paris (H. Brezis and J. L. Lions, eds.), Vol. 6, Pitman, 1984.

[GLM1] S. Granlund, P. Lindquist and O. Martio, Conformally invariant variational integrals, Trans. Amer. Math. Soc. 277 (1983), 43-73.

[GLM2] _ Note on the PW B-method in the non-linear case, Pacific J. Math. 125 (1986), 381. 395.

[HW] L. I. Hedberg and Th. H. Wolff, Thin sets in nonlinear potential theory, Ann. Inst. Fourier (Grenoble) 33 (1983), $161-187$.

[HK1] J. Heinonen and T. Kilpeläinen, A-superharmonic functions and supersolutions of degenerate elliptic equations, Ark. Mat. (to appear).

[HK2] _ Polar sets for supersolutions of degenerate elliptic equations, Math. Scand. (to appear).

[KS] D. Kinderlehrer and G. Stampacchia, An introduction to variational inequalities and their applications, Academic Press, New York, 1980.

[LS] H. Lewy and G. Stampacchia, On the regularity of the solution of a variational inequality, Comm. Pure Appl. Math. 22 (1969), 153 188. 
[L] P. Lindqvist, On the definition and properties of p-superharmonic functions, J. Reine Angew. Math. 365 (1986), 67-79.

[LM] P. Lindqvist and O. Martio, Two theorems of N. Wiener for solutions of quasilinear elliptic equations, Acta Math. 155 (1985), 153-171.

[M1] V. G. Maz'ya, On the continuity at a boundary point of solutions of quasi-linear elliptic equations, Vestnik Leningrad Univ. Mat. Mech. Astronom. 13 (1970), 42-55; English transl., Vestnik Leningrad Univ. Math. 3 (1976), 225-242.

[M2] _ Sobolev spaces, Springer-Verlag, New York and Berlin, 1985.

[MK] V. G. Maz'ya and V. P. Khavin, Non-linear potential theory, Russian Math. Surveys 27 (1972), 71-148.

[Me] N. G. Meyers, Continuity properties of potentials, Duke Math. J. 42 (1975), 157-166.

[MZ] J. H. Michael and W. P. Ziemer, Interior regularity for solutions to obstacle problems, Nonlinear Analysis 10 (1986), 1427-1448.

[Mo1] U. Mosco, Wiener criterion and potential estimates for the obstacle problem, Indiana Univ. Math. J. 36 (1987), 455-494.

[Mo2] _ Wiener criteria and variational convergences, SFB 72, Preprint 797, Bonn, 1986.

[R] Yu. G. Reshetnyak, The concept of capacity in the theory of functions with generalized derivatives, Sibirsk. Mat. Zh. 10 (1969), 1109-1138 (Russian).

[Sa] J. Sarvas, Symmetrization of condensers in n-space, Ann. Acad. Sci. Fenn. Ser. A I Math. 522 (1972), 1-44.

DEPARTMENT OF MATHEMATICS, UNIVERSity OF JyVÄSKYLÄ, SEMinaARINKATU 15, SF-40100 JYVÄSKYLÄ, FINLAND (Current address of Tero Kilpeläinen)

INSTITUT FÜR ANGEWANDTE MATHEMATIK DER UNIVERSITÄt BONN, SONDERFORSCHUNGSBereich 256, Wegelerstrasse 6, D-5300 BonN 1, Federal Republic of GERMANY

Current address (Juha Heinonen): Department of Mathematics, University of Michigan, Ann Arbor, Michigan 48109-1003 